\title{
Inhibition of miR-101-3p protects against sepsis-induced myocardial injury by inhibiting MAPK and NF-кB pathway activation via the upregulation of DUSP1
}

\author{
YE XIN $^{1}$, LI TANG $^{2}$, JING CHEN $^{1}$, DONG CHEN $^{1}$, WEN WEN $^{1}$ and FUGANG HAN ${ }^{1}$ \\ ${ }^{1}$ Department of Radiology, The Affiliated Hospital of Southwest Medical University, Luzhou, Sichuan 646000; \\ ${ }^{2}$ Department of Gastroenterology, Xinqiao Hospital of Army Medical University, Chongqing 400037, P.R. China
}

Received May 20, 2020; Accepted October 5, 2020

DOI: $10.3892 / \mathrm{ijmm} .2021 .4853$

\begin{abstract}
Numerous studies have found that microRNAs (miRNAs or miRs) are aberrantly expressed when sepsis occurs. The present study aimed to investigate the role of miR-101-3p in sepsis-induced myocardial injury and to elucidate the underlying mechanisms. Models of myocardial injury were established both in vivo and in vitro. The results revealed that miR-101-3p was upregulated in the serum of patients with sepsis-induced cardiomyopathy (SIC) and positively correlated with the levels of pro-inflammatory cytokines (including IL-1 $\beta$, IL- 6 and TNF- $\alpha$ ). Subsequently, rats were treated with miR-101-3p inhibitor to suppress miR-101-3p and were then exposed to lipopolysaccharide (LPS). The results revealed that LPS induced marked cardiac dysfunction, apoptosis and inflammation. The inhibition of miR-101-3p markedly attenuated sepsis-induced myocardial injury by attenuating apoptosis and the expression of pro-inflammatory cytokines. Mechanistically, dual specificity phosphatase-1 (DUSP1) was found to be a functional target of miR-101-3p. The downregulation of miR-101-3p led to the overexpression of DUSP1, and the inactivation of the MAPK $\mathrm{p} 38$ and NF- $\mathrm{KB}$ pathways. Moreover, blocking DUSP1 by short hairpin RNA against DUSP1 (sh-DUSP1) significantly reduced the myocardial protective effects mediated by the inhibition of miR-101-3p. Collectively, the findings of the present study demonstrate that the inhibition of miR-101-3p exerts cardioprotective effects by suppressing MAPK p38 and NF- $\mathrm{KB}$ pathway activation, and thus attenuating inflammation and apoptosis dependently by enhancing DUSP1 expression.
\end{abstract}

Correspondence to: Dr Fugang Han, Department of Radiology, The Affiliated Hospital of Southwest Medical University, 25 Taiping Street, Jiangyang, Luzhou, Sichuan 646000, P.R. China E-mail: fuganghan92@163.com

Key words: sepsis, myocardial injury, miR-101-3p, dual specificity phosphatase-1, inflammation

\section{Introduction}

Myocardial injury is a common complication of sepsis, and is independently associated with early, but not late mortality and post-discharge cardiovascular morbidity (1). The pathological manifestations of sepsis-induced cardiomyopathy (SIC) are as follows: Spot hemorrhage on the myocardial surface, extensive myocardial hemorrhage, inflammatory cell infiltration, interstitial edema and degeneration and necrosis of some myocardial cells (2-4). During the process of sepsis, bacterial infection activates Toll-like receptor (TLR) via pathogen-related molecular patterns, such as lipopolysaccharide (LPS), thereby inducing MAPK and NF- $\mathrm{KB}$ pathway activation, which promotes the infiltration of inflammatory cells into myocardial tissue and triggers myocardial inhibition (5-9). Therefore, it is currently hypothesized that the inhibition of the NF- $\mathrm{KB}$ and MAPK pathways can effectively reduce the inflammatory response of the septic myocardium and is one of the strategies used to improve SIC.

Dual specificity phosphatase-1 (DUSP1) is the first member of the MAP kinase phosphatase (MKP) family, with a size of $1101 \mathrm{bp}$ and encoding 367 amino acids. MKP is a member of the bidirectional specificity Sue/tyrosine phosphatase family and is comprised of 25 members, which results in both serine/threonine phosphorylation and tyrosine phosphorylation $(10,11)$. Studies have demonstrated that DUSP1 can regulate the MAPK signaling pathway by regulating the dephosphorylation of threonine/serine and tyrosine residues and regulating cell proliferation, division and cytokine production $(12,13)$. Additionally, DUSP1 can act as a direct target of p53, transcription factor E2F1, c-Jun and cyclic AMP-dependent transcription factor ATF2 during sepsis, ischemia, hypoxia, oxidative stress and other environmental effects $(14,15)$.

MicroRNAs (miRs/miRNAs) are a type of endogenous non-coding single-stranded small RNA with a length of 19-23 nucleotide sequences. They are highly conserved in evolution, mainly through full or partial matching with the 3 '-untranslated region (3'-UTR) of target mRNAs to regulate physiological or pathological processes in human genes (16). In recent years, an increasing number of studies have demonstrated that miRNAs, such as miR-146b (17), play an important role in the diagnosis 
and treatment of SIC. Additionally, miR-101-3p, which is an active form of miR-101, is widely found in eukaryotic cells and can be abnormally expressed as a tumor suppressor in gastric cancer (18) and breast cancer (19). In non-tumor diseases, miR-101-3p has been shown to be significantly upregulated in the plasma of patients with adult onset Still's disease (AOSD) and to positively correlate with the expression of inflammatory mediators (20). Moreover, miR-101-3p also affects other inflammation-related diseases, such as rheumatoid arthritis (21) and heart transplant recipients with histologically-verified acute cellular rejection (22). However, to the best of our knowledge, there are limited studies available investigating the effects of miR-101-3p on the inflammatory response post-SIC.

Therefore, the present study aimed to further explore the effects of miR-101-3p in SIC and its possible mechanisms both in vitro and in vivo.

\section{Materials and methods}

Patient data. A total of 27 patients with SIC admitted to the Affiliated Hospital of Southwest Medical University between January, 2017 to January, 2019 were selected as the study subjects. Subjects included 16 males and 11 females, aged 21-56 years, with an average age of $36.4 \pm 8.4$ years. A total of 15 healthy volunteers enrolled at the same hospital during the same time period were included as the control group, including 10 males and 5 females, aged 21-56 years, with an average age of $35.7 \pm 8.3$ years. The exclusion criteria were as follows: Pregnant females, organ transplant recipients and acquired immunodeficiency syndrome. Prior to the study, all participants signed informed consent forms and agreed to the use of their samples in this scientific research. Venous blood was obtained from all patients at the time of diagnosis, and cardiac ultrasound was performed to examine cardiac function within $24 \mathrm{~h}$ following diagnosis. The present study was approved by the Ethics Committee of the Affiliated Hospital of Southwest Medical University.

Establishment of an animal model of SIC and drug administration. Sprague-Dawley male rats, weighing 220-250 g, were purchased from the Nanjing Experimental Animal Center. All rats were raised in a standard environment, and there were no marked differences in the age and weight between the rats. The Sprague-Dawley rats were randomly divided into the sham-operated (sham) group $(n=15)$ and the LPS group ( $20 \mathrm{mg} / \mathrm{kg}$ group; $\mathrm{n}=15)$. The model of SIC was established according to a previous study (23). Briefly, the rats in each group were intraperitoneally injected with the corresponding dose of LPS (20 mg/kg, Sigma-Aldrich; Merck $\mathrm{KGaA}$ ) (23) or the same volume of saline. After $6 \mathrm{~h}$, the cardiac function of each group of rats was detected by ultrasound. The sepsis model was confirmed by the following: i) Clinical signs, such as featuring malaise, fever, chills, piloerection, generalized weakness and reduced gross motor activity; ii) cardiac function examination by echocardiography showing a reduced ejection fraction $[\mathrm{EF}(\%)]$ and fractional shortening [FS (\%)]; iii) significantly increased levels of pro-inflammatory cytokines in serum. miR-101-3p inhibitors were obtained from Guangzhou RiboBio Co., Ltd. For the downregulation of miR-101-3p, miR-101-3p inhibitors (10 nmol) or negative controls (miR-101-3p NC, $10 \mathrm{nmol}$ ) in $50 \mu \mathrm{l}$ PBS were administered to the rats $(n=20$ in each group) via the caudal vein once every 3 days (normal saline was used as a control) prior to LPS stimulation. The animal experiments were approved by the Ethics Committee of the Affiliated Hospital of Southwest Medical University. All experimental procedures were performed in accordance with the National Institutes of Health Guidelines for the Care and Use of Laboratory Animals.

Examination of cardiac function. Echocardiography was performed using a Mylab $30 \mathrm{CV}$ ultrasound system (Esoate, S.P.A.) and a $10 \mathrm{mHz}$ linear ultrasound sensor. The procedure is briefly described as follows: Following anesthesia [via an intraperitoneal injection of pentobarbital sodium $(50 \mathrm{mg} / \mathrm{kg}$ body weight)], the rats were shaved in the anterior chest area and placed on a heating plate at $37^{\circ} \mathrm{C}$ with the left side up. The probe was applied with a coupling agent and pressed against the chest wall, and indexes, including EF (\%), FS (\%) and heart rate were detected.

Cell culture and treatment. H9C2 human cardiomyocytes were obtained from the Cell Bank of the Chinese Academy of Sciences. H9C2 cells were cultured in DMEM (Invitrogen; Thermo Fisher Scientific, Inc.) supplemented with L-glutamine (Gibco; Thermo Fisher Scientific, Inc.), $100 \mathrm{U} / \mathrm{ml}$ penicillin, $100 \mathrm{mg} / \mathrm{ml}$ Sinopharm Chemical Reagent and 10\% FBS (Gibco; Thermo Fisher Scientific, Inc.). The cells were incubated in a humidified incubator at $37^{\circ} \mathrm{C}$ with $5 \% \mathrm{CO}_{2} . \mathrm{H} 9 \mathrm{C} 2$ cells were treated with $10 \mu \mathrm{g} / \mathrm{ml}$ LPS (Sigma-Aldrich; Merck $\mathrm{KGaA}$ ) for $24 \mathrm{~h}$ to establish an in vitro model of LPS-induced sepsis.

Cell transfection. $\mathrm{H} 9 \mathrm{C} 2$ cells were inoculated in a 6-well plate at a density of $5 \times 10^{5}$ cells $/ \mathrm{ml}$ and cultured at $37^{\circ} \mathrm{C}$ with $5 \% \mathrm{CO}_{2}$ for $24 \mathrm{~h}$ prior to transfection. miR-101-3p NC, miR-101-3p inhibitors and short hairpin RNA (sh)-DUSP1 were purchased from Guangzhou RiboBio Co., Ltd. The H9C2 cells were transfected with the above-mentioned vectors $(10 \mathrm{nmol} / \mathrm{ml})$ using Lipofectamine ${ }^{\circledR} 3000$ (Invitrogen; Thermo Fisher Scientific, Inc.) according to the manufacturer's instructions. The transfection efficiency was measured by reverse transcription-quantitative PCR (RT-qPCR). Following $48 \mathrm{~h}$ of transfection, the cells were further cultured with fresh medium at $37^{\circ} \mathrm{C}$ and $5 \% \mathrm{CO}_{2}$.

$R T-q P C R$. Total RNA was extracted from the myocardial tissues and H9C2 cells using TRIzol ${ }^{\circledR}$ reagent (Invitrogen; Thermo Fisher Scientific, Inc.). Reverse transcription was performed using MMLV reverse transcriptase (Invitrogen; Thermo Fisher Scientific, Inc.) to generate first-strand cDNA. The ABI 7500 Real-Time PCR system (Applied Biosystems; Thermo Fisher Scientific, Inc.) and SYBR premix Ex Taq II (Takara Biotechnology Co.,Ltd.) was used to perform RT-qPCR according to the manufacturer's instructions. The following thermocycling conditions were used for qPCR: Pre-denaturation at $95^{\circ} \mathrm{C}$ for $10 \mathrm{~min}$, and 45 cycles of $95^{\circ} \mathrm{C}$ for $15 \mathrm{sec}$ and $60^{\circ} \mathrm{C}$ for $15 \mathrm{sec}$. Fluorescent signals were obtained at $60^{\circ} \mathrm{C}$. GAPDH was used as the internal reference gene for DUSP1, IL-1 $\beta$, IL-6 and TNF- $\alpha$ expression. U6 was used as standardized internal reference of $\mathrm{miR}-101-3 \mathrm{p}$ expression. Gene expression 
was calculated using the $2^{-\Delta \Delta \mathrm{Cq}}$ method (24). Each experiment was repeated and measured 3 times. Primers were designed using Primer 6.0 software based on the serial number of each gene in GenBank. The following primer pairs were used for qPCR: $\alpha$-MHC forward, 5'-GCCTCTCTGATGGTTCAGC-3' and reverse, 5'-TTAGAATTTGCTTTGGGTG-3'; $\beta$-MHC forward, 5'-TGATACGGCCGTGCCTCT-3' and reverse, 5'-AGCTCTACGATTGTGGCATGCT-3'; S100A8 forward, 5'-CCGTCAGCCTGGTTGCTC-3' and reverse, 5'-CTTCAC CAGTCGACGATG-3'; S100A9 forward, 5'-GATCGCCCT CTCTGTTTAAAGC-3' and reverse, 5'-ATCCCACCTTAT GTGTCCTTGG-3'; DUSP1 forward, 5'-ATATGTTGGAGG GAGACGAC-3' and reverse, 5'-ACAGAAGCCTGTTCCTGG TGT-3'; TNF- $\alpha$ forward, 5'-GACCCCTTTACTCTGACCCC-3' and reverse, 5'-AGGCTCCAGTGAATTCGGAA-3'; IL-1 $\beta$ forward, 5'-ACAGATGAAGTGCTCCTTCCA-3' and reverse, 5'-GTCGGAGATTCGTAGCTGGA-3'; IL-6 forward, 5'-TGT CTTCCTCACCGATTCCT-3' and reverse, 5'-ACCACCCGA GCTCTGTCTTACTC-3'; GAPDH forward, 5'-TATGATGAT ATCAAGAGGGTAGT-3' and reverse, 5'-TGTATCCAAACT CATTGTCATAC-3'; miR-101-3p forward, 5'-GGACTTTCT TCATTCACACCG-3' and reverse, 5'-GACCACTGAGGTTAG AGCCA-3' and U6 forward, 5'-GCTTCGGCAGCACATATA CTAAAAT-3' and reverse, 5'-CGCTTCACGAATTTGCGT GTCAT-3'.

ELISA. ELISA kits (Wuhan Boster Biological Technology, Ltd.) were used to detect the levels of the inflammatory factors, IL-1 $\beta$, IL- 6 and TNF- $\alpha$, in the serum of patients with SIC or healthy controls (HCs) according to the manufacturer's protocols. The measured optical density value (at a wavelength of $450 \mathrm{~nm}$ ) was converted to the concentration value through the Multiskan FC Microplate Reader (Thermo Fisher Scientific, Inc.). The experiment was repeated 3 times.

Western blot analysis. The undetermined cells were washed 3 times with PBS, and total protein was extracted using RIPA buffer (Beyotime Institute of Biotechnology, Inc.) and protease inhibitor PMSF or cocktail (Roche Diagnostics). The concentration of total protein was determined by the BSC method (Beyotime Institute of Biotechnology, Inc.). Proteins were denatured at $100^{\circ} \mathrm{C}$ for $5 \mathrm{~min}$. Equal amounts of protein $(50 \mu \mathrm{g})$ were separated by $10 \%$ SDS-PAGE and transferred to PVDF membranes. Subsequently, PVDF membranes were blocked with $5 \%$ skim milk for $2 \mathrm{~h}$. The membranes were incubated with anti-MKP-1 (DUSP1) antibody (cat. no. ab1351; 1:1,000; Abcam), anti-p38 antibody (cat. no. ab31828; 1:1,000; Abcam), anti-phosphorylated-p38 antibody (cat. no. ab178867; 1:1,000; Abcam), anti-NF- $\mathrm{B}$ p65 antibody (cat. no. ab140751; 1:1,000; Abcam), anti-phosphorylated-NF- $\mathrm{B}$ p65 antibody (cat. no. ab76302; 1:1,000; Abcam) and anti-GAPDH antibody (cat. no. ab9485; 1:2,000; Abcam) overnight at $4^{\circ} \mathrm{C}$. Following washing, membranes were incubated with corresponding secondary antibodies [goat anti-rabbit IgG H\&L (HRP), cat. no. ab205718, 1:2,000, Abcam; goat anti-mouse IgG H\&L (HRP), cat. no. ab6789, 1:2,000, Abcam] at room temperature for $2 \mathrm{~h}$. Protein bands were visualized with drip developer development (GS-700, Bio-Rad Laboratories, Inc.), and Molecular Analyst (IBM, Inc.) was used for densitometry. The experiment was repeated 3 times.
Luciferase reporter gene assay. The coding sequence of human DUSP1 3'-UTR was obtained from GenBank and analyzed by bioinformatics analysis. The potential binding sites between miR-101-3p with DUSP1 was analyzed using TargetScan 6.0 (http://www.targetscan.org). The wild-type DUSP1 (DUSP1-WT) 3'-UTR fragment and the mutant-type DUSP1 (DUSP1-MUT) 3'-UTR fragment were cloned into a pGL3-control vector downstream (GeneCopoeia, Inc.) of the luciferase coding sequence to obtain LuDP recombinant plasmids. H9C2 cells were inoculated into 96-well plates and transfected with miR-101-3p mimics or miR-101-3p NC or DUSP1-WT plasmids or DUSP1-MUT plasmids (100 nM aliquots), respectively. Lipofectamine ${ }^{\circledR} 3000$ (Invitrogen; Thermo Fisher Scientific, Inc.) was used as transfection reagent. The luciferase activity of the cells was measured at $24 \mathrm{~h}$ following transfection using a dual luciferase reporter gene system (Promega Corporation). The experiment was repeated 3 times, and the blank group was used for normalization.

Hematoxylin and eosin $(H \& E)$ staining and immunohistochemistry (IHC). After $6 \mathrm{~h}$ of LPS stimulation, the rats in each group were sacrificed using Nembutal (intraperitoneal sodium pentobarbital; $80 \mathrm{mg} / \mathrm{kg} /$ body weight). Subsequently, the rats underwent cardiac perfusion with at least $100 \mathrm{ml}$ saline, and heart tissues were obtained, fixed with paraformaldehyde and embedded in paraffin.

H\&E staining was then performed to assess pathological changes. Briefly, the tissues were continuously sectioned into coronary sections of approximately $5 \mu \mathrm{m}$ in thickness. The sections were then routinely dewaxed with xylene and hydrated with gradient alcohol and finally rinsed with distilled water. They sections were then immersed in hematoxylin dye solution for 3-8 min and washed at room temperature, and then differentiated by $1 \%$ hydrochloric acid alcohol for several seconds, then washed with water. After washing for 10-15 min using a small water flow, the slices were placed into eosin dye solution for 1-3 min at room temperature. The slices were then dehydrated and transparent by alcohol and xylene for $5 \mathrm{~min}$ each, and the slices were then dried and sealed with neutral gum. A microscope (Zeiss AxioImager Z1) was used for observation and image analyses were conducted.

Additionally, IHC was performed to detect caspase 3-and TUNEL-labeled apoptotic cells and DUSP1 expression in heart tissues according to a previous study (25). Briefly, the tissues were continuously sectioned into coronary sections of approximately $5 \mu \mathrm{m}$ in thickness. Subsequently, the sections were routinely dewaxed with xylene and hydrated with gradient alcohol. After the sections were blocked with $3 \%$ $\mathrm{H}_{2} \mathrm{O}_{2}$ for $10 \mathrm{~min}$, the endogenous peroxidase was inactivated, and $0.01 \mathrm{~mol} / 1$ sodium citrate buffer was used for microwave

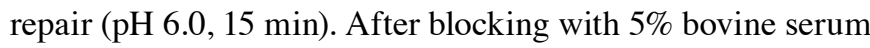
albumin (BSA) for $20 \mathrm{~min}$, the primary antibodies were added and incubated with the sections overnight at $4^{\circ} \mathrm{C}$. On the following day, goat anti-rabbit IgG H\&L (HRP) (ab6721, 1:100, Abcam) was added and incubated with the sections at room temperature for $60 \mathrm{~min}$, and the sections were then developed with DAB after being washed with PBS. Following hematoxylin counterstaining at room temperature for 5-8 min, the sections were dehydrated and transparentized, and mounted for microscopic examination (Zeiss AxioImager Z1). 



Figure 1. Expressional characteristics of miR-101-3p in patients with SIC and its correlation with pro-inflammatory factors. (A) miR-101-3p expression in patients with SIC and healthy controls was detected using reverse transcription-quantitative PCR. (B) Serum IL-1 $\beta$, IL- 6 and TNF- $\alpha$ levels were detected using ELISA. (C-E) Correlation analysis was used to analyse the correlation between miR-101-3p expression with IL-1 $\beta$, IL- 6 and TNF- $\alpha$. ${ }^{*} \mathrm{P}<0.05$; *** $\mathrm{P}<0.01$ and ${ }^{* * *} \mathrm{P}<0.001$. miR, microRNA; SIC, sepsis-induced cardiomyopathy.

Image-Pro Plus image analysis software (MediaCybemetics, Inc.) was employed to analyze the number of positive cells. The following primary antibodies were used: Anti-caspase 3 (cat. no. ab4051; 1:200; Abcam) and anti-MKP-1 (DUSP1; cat. no. ab1351; 1:100; Abcam). The TUNEL Assay kit was also used (cat. no. ab206386; Abcam).

Statistical analysis. SPSS 21.0 was used for statistical analysis (IBM Corp.). Data are expressed as the means \pm SD. A Student's t-test was used to compare differences between 2 groups, and one-way ANOVA was used for the analysis of multiple groups, followed by Tukey's post hoc test. Pearson's correlation analysis was performed to determine the correlation between cytokine levels and miR-101-3p expression in tissues from different patients or rats. $\mathrm{P}<0.05$ was considered to indicate a statistically significant difference.

\section{Results}

miR-101-3p expression is increased in patients with SIC. To preliminarily assess he expression of miR-101-3p in SIC, the expression levels of miR-101-3p in the serum of patients with SIC and HCs were detected. The results revealed that miR-101-3p expression in the serum of patients with SIC was significantly upregulated (Fig. 1A). Moreover, the expression of the pro-inflammatory factors, IL- $1 \beta$, IL- 6 , and TNF- $\alpha$, in serum was detected by EILSA. The results revealed that the expression levels of the pro-inflammatory factors, IL-1 $\beta$, IL-6, and TNF- $\alpha$, were all significantly upregulated in the serum of patients with SIC compared with the HCs (Fig. 1B). Further analysis revealed that there was a significant positive correlation between miR-101-3p, and IL-1 $\beta$, IL-6 and TNF- $\alpha$ expression $(\mathrm{P}<0.05$; Fig. $1 \mathrm{C}-\mathrm{E})$. These data indicated 


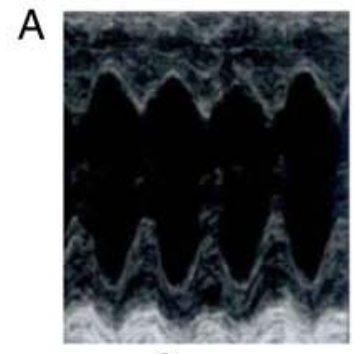

Sham

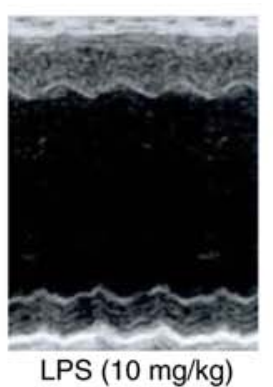

LPS (10 mg/kg)
B

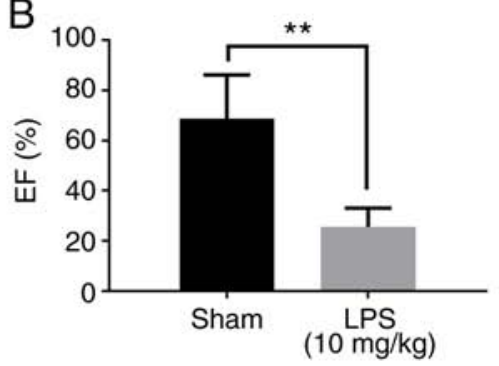

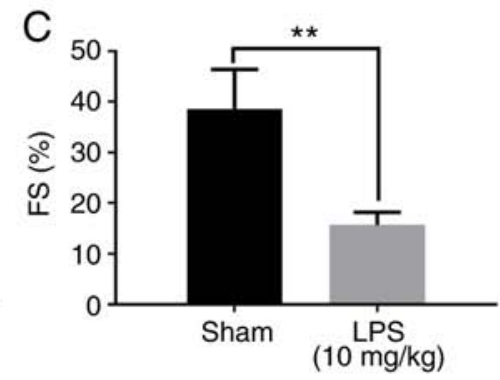

D

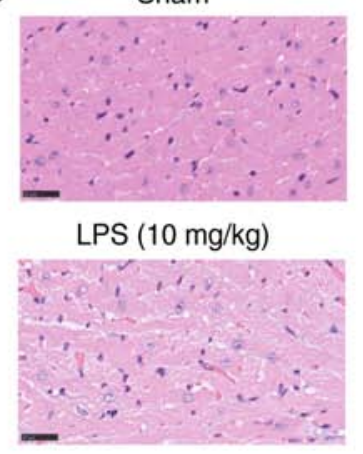

E

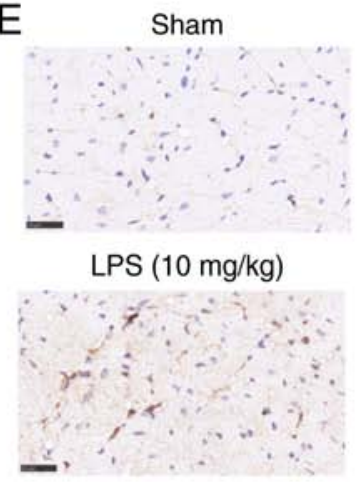

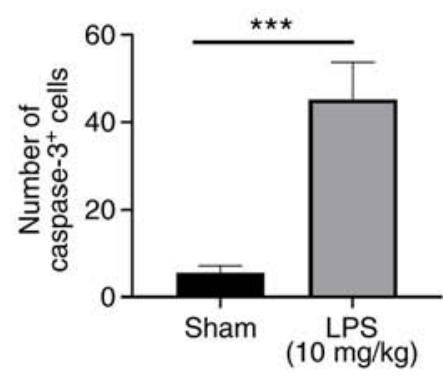

F

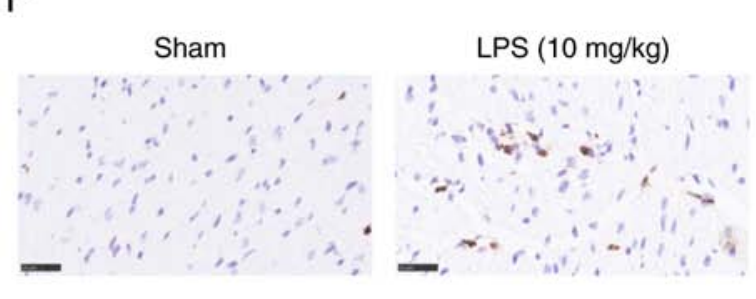

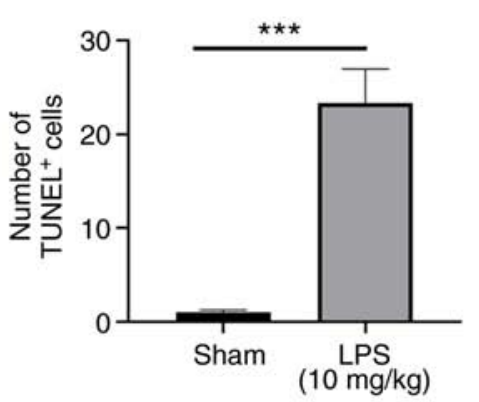

G

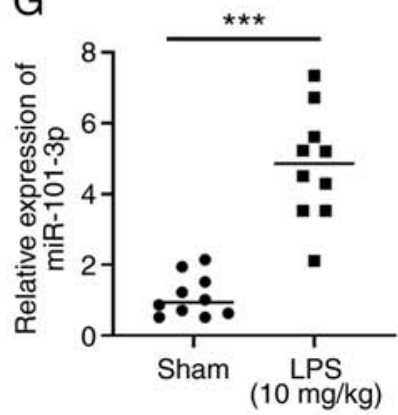

Figure 2. Expression of miR-101-3p in myocardial tissues in a rat model of sepsis-induced MI. LPS $(20 \mathrm{mg} / \mathrm{kg})$ was administered to rats to establish a model of sepsis-induced MI. (A-C) Ultrasound was used to evaluate rat cardiac functions. The EF (\%) and FS (\%) were measured. (D and E) H\&E staining, IHC and RT-qPCR were used to detect the (D) histopathological changes of cardiac tissues, and (E) caspase 3- and (F) TUNEL-labeled cardiac apoptotic cells. (G) miR-101-3p expression in the myocardial tissue of rats was detected by RT-qPCR. ${ }^{* *} \mathrm{P}<0.01$ and ${ }^{* * *} \mathrm{P}<0.001$. miR, microRNA; MI, myocardial injury; LPS, lipopolysaccharide; EF, ejection fraction; FS, fractional shortening; H\&E, hematoxylin and eosin; IHC, immunohistochemistry; RT-qPCR, reverse transcription-quantitative PCR.

that miR-101-3p may play a role in SIC by modulating inflammation.

Upregulation of miR-101-3p expression in the myocardial tissue of rats administered LPS. To further investigate the role of miR-101-3p in sepsis-induced myocardial injury, a rat model of SIC was established. Ultrasound was used to evaluate the cardiac function of the rats. The results revealed that the EF (\%) and FS (\%) of the rats in the LPS group were significantly lower compared with those of the rats in the sham group $(\mathrm{P}<0.05$; Fig. $2 \mathrm{~A}-\mathrm{C})$. Moreover, the results of $\mathrm{H} \& \mathrm{E}$ staining and $\mathrm{IHC}$ indicated that there was significant myocardial cell injury, cell apoptosis and inflammatory cell infiltration in the rats in the LPS group (Fig. 2D-F). These data confirmed that the model of SIC was successfully established. Furthermore, miR-101-3p expression in the rat myocardial tissues was detected. The results revealed that compared with the sham group, the miR-101-3p levels in the LPS group were significantly increased ( $\mathrm{P}<0.05$; Fig. $2 \mathrm{G})$. Therefore, the aforementioned data indicated that miR-101-3p plays a role in the process of sepsis-induced myocardial injury.

Inhibition of miR-101-3p significantly attenuates LPS-induced myocardial injury. To evaluate the effects of miR-101-3p on the cardiac function of rats with sepsis-induced myocardial injury, the rats were treated with miR-101-3p inhibitors to inhibit miR-101-3p expression. The results of RT-qPCR revealed that $\mathrm{miR}-101-3 \mathrm{p}$ expression was significantly downregulated following treatment with miR-101-3p inhibitors compared with the LPS + saline or miR-101-3p NC group (Fig. 3A). Further assessment of the cardiac function of the rats revealed that the inhibition of miR-101-3p significantly enhanced the EF (\%) and FS (\%) compared with the LPS + saline or miR-101-3p NC group (Fig. 3B and C). Histopathological analysis revealed that the downregulation of miR-101-3p suppressed cardiac cell damage, inflammatory cell infiltration 

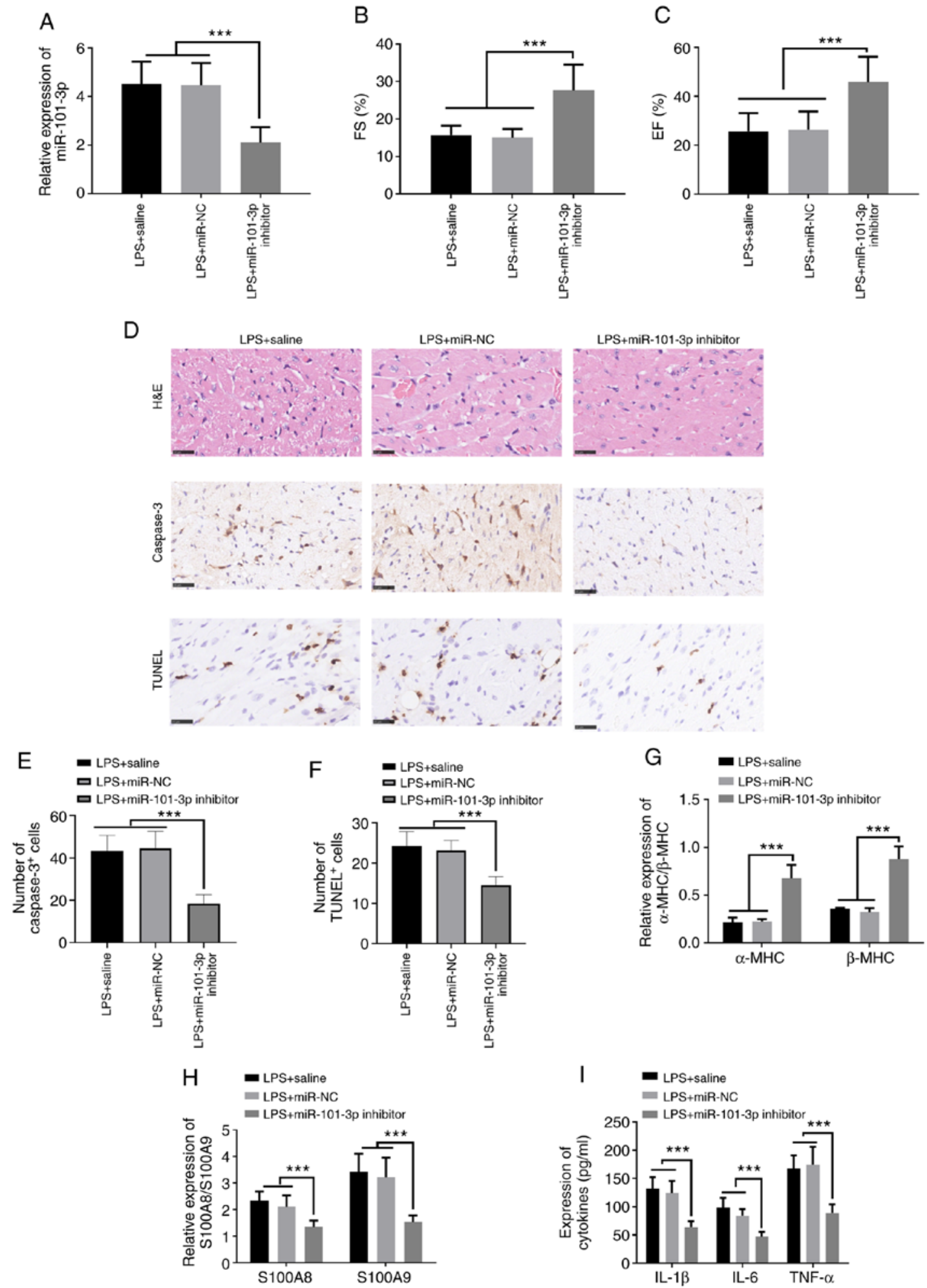

Figure 3. Regulatory effects of miR-101-3p on cardiac function in rats with LPS-induced SIC. miR-101-3p inhibitors (10 nmol) or negative controls (miR-101-3p $\mathrm{NC}$; $10 \mathrm{nmol}$ ) in $50 \mu \mathrm{l}$ PBS were administered into the caudal veins of rats once for 3 days, and were then treated with LPS (20 mg/kg) for $6 \mathrm{~h}$. (A) The expression levels of miR-101-3p was detected using RT-qPCR. (B and C) Ultrasound was used to evaluate rat cardiac function. The EF (\%) and FS (\%) were measured. (D and E) H\&E staining, IHC and RT-qPCR were used to detect (D) the histopathological changes of cardiac tissues and (E and F) the apoptosis of cardiac cells. (G and H) Differential expression of $\alpha$-MHC, $\beta$-MHC, S100A8 and S100A9 in the myocardial tissues of different groups of rats detected by RT-qPCR. (I) Differential expression of IL-1 $\beta$, IL-6, and TNF- $\alpha$ in the myocardial tissues of rats in different groups detected using ELISA. ${ }^{* * * *} \mathrm{P}<0.001$. miR, microRNA; LPS, lipopolysaccharide; SIC, sepsis-induced cardiomyopathy; EF, ejection fraction; FS, fractional shortening; H\&E, hematoxylin and eosin; IHC, immunohistochemistry; RT-qPCR, reverse transcription-quantitative PCR; MHC, myosin heavy chain.

and apoptosis (Fig. 3D-F). In addition, RT-qPCR was used to detect changes in the expression of a-myosin heavy chain $(\alpha-\mathrm{MHC})$ and $\beta$-MHC in myocardial tissues. Following the addition of miR-101-3p inhibitors, the $\alpha$-MHC and $\beta$-MHC levels were significantly upregulated, while those of S100A8 and S100A9 were significantly downregulated compared 


\section{A DUSP1WT: 5' UUUUGUUUUUG-AGUACUGUA $3^{\prime}$ \\ I II I IIIIIIII \\ miR-101-3p: 3' AAGUCAAUAGUGUCAUGACAU 5'}

DUSP1 MT: 5' UUUUCAAUUUG-UGAAGACAA 3'

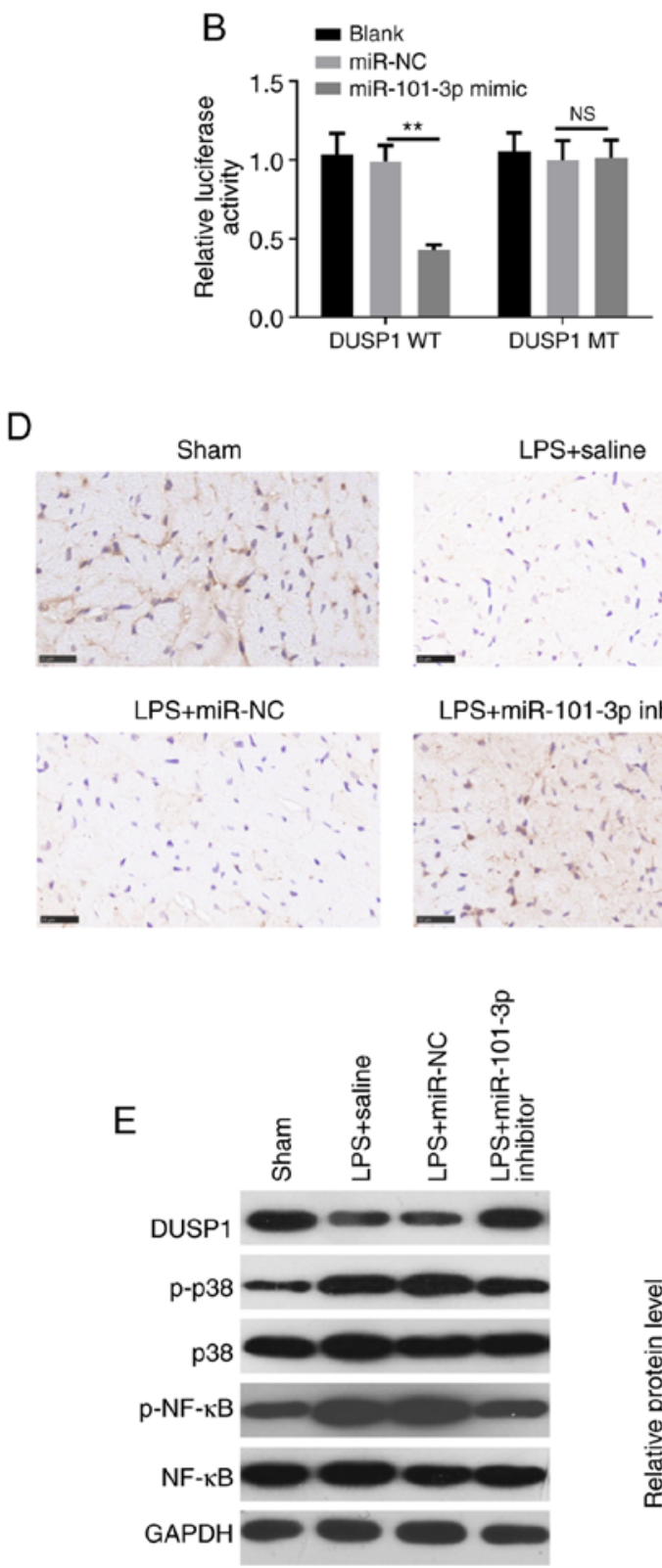

C
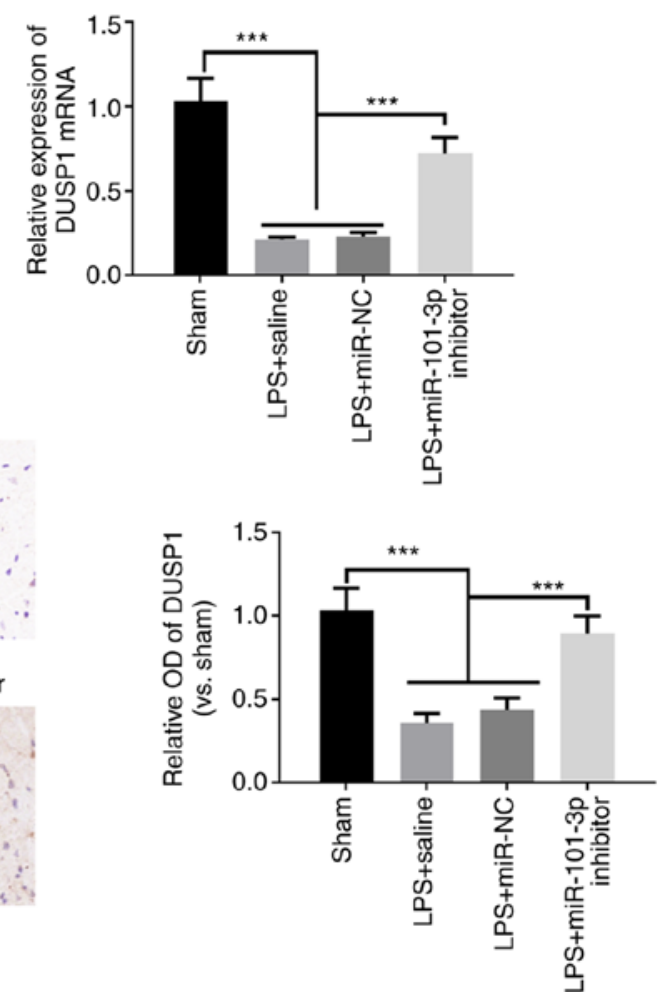

Figure 4. DUSP1 is a functional target of miR-101-3p. (A) TargetScan7.1 was used to analyze the downstream targets of miR-101-3p. The results revealed that DUSP1 was one of the targets of miR-101-3p. (B) Luciferase activity assay was performed to detect changes in luciferase activity after interaction of miR-101-3p mimics and DUSP1 wild type (DUSP1-WT) and DUSP1 mutant (DUSP1-MT). (C) Reverse transcription-quantitative PCR and (D) immunohistochemistry was used to detect DUSP1 expression in cardiac tissues. (E) Western blot analysis was conducted to assess the levels of DUSP1, MAPK-p38 and NF-kB in myocardial tissue. NS, not significant $(\mathrm{P}>0.05) ;{ }^{* * *} \mathrm{P}<0.001$. miR, microRNA; DUSP1, dual specificity phosphatase 1.

with the LPS + saline or miR-101-3p NC groups $(\mathrm{P}<0.05$; Fig. $3 \mathrm{G}$ and $\mathrm{H})$. ELISA was also used to detect the expression of IL-1 $\beta$, IL- 6 and TNF- $\alpha$. The expression levels of IL-1 $\beta$, IL- 6 and TNF- $\alpha$ were significantly downregulated following the addition of miR-101-3p inhibitor compared with the LPS + saline or miR-101-3p NC groups $(\mathrm{P}<0.05$; Fig. 3I). Taken together, these data indicated that the downregulation of miR-101-3p exerted protective effects against sepsis-induced myocardial injury by mitigating inflammation and apoptosis.
DUSP1 is a functional target of miR-101-3p. To verify the specific mechanisms through which miR-101-3p improves LPS-induced myocardial function in rats, the downstream targets of miR-101-3p were analyzed using TargetScan 7.1 (http://www.targetscan.org/vert_71/). The results revealed that DUSP1 was one of the targets of miR-101-3p (Fig. 4A). To further validate the targeting association between miR-101-3p and DUSP1, a luciferase activity assay was performed. The results demonstrated that compared with the control group, 

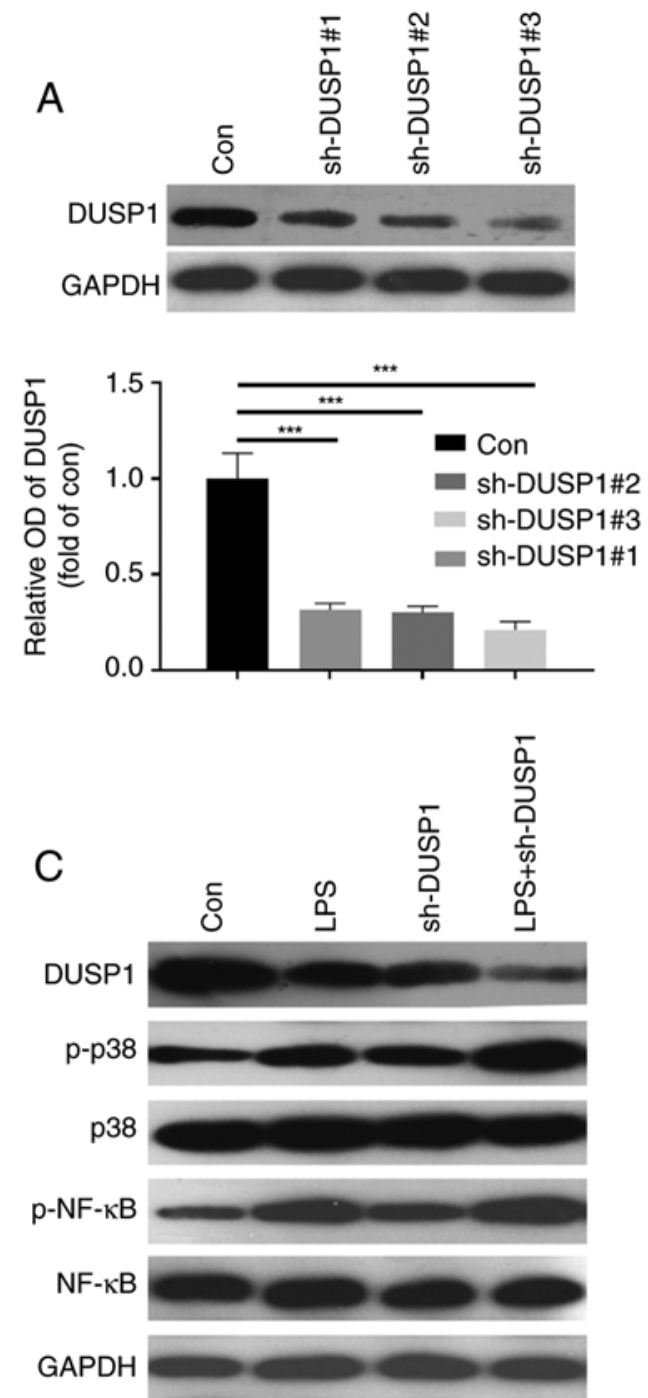
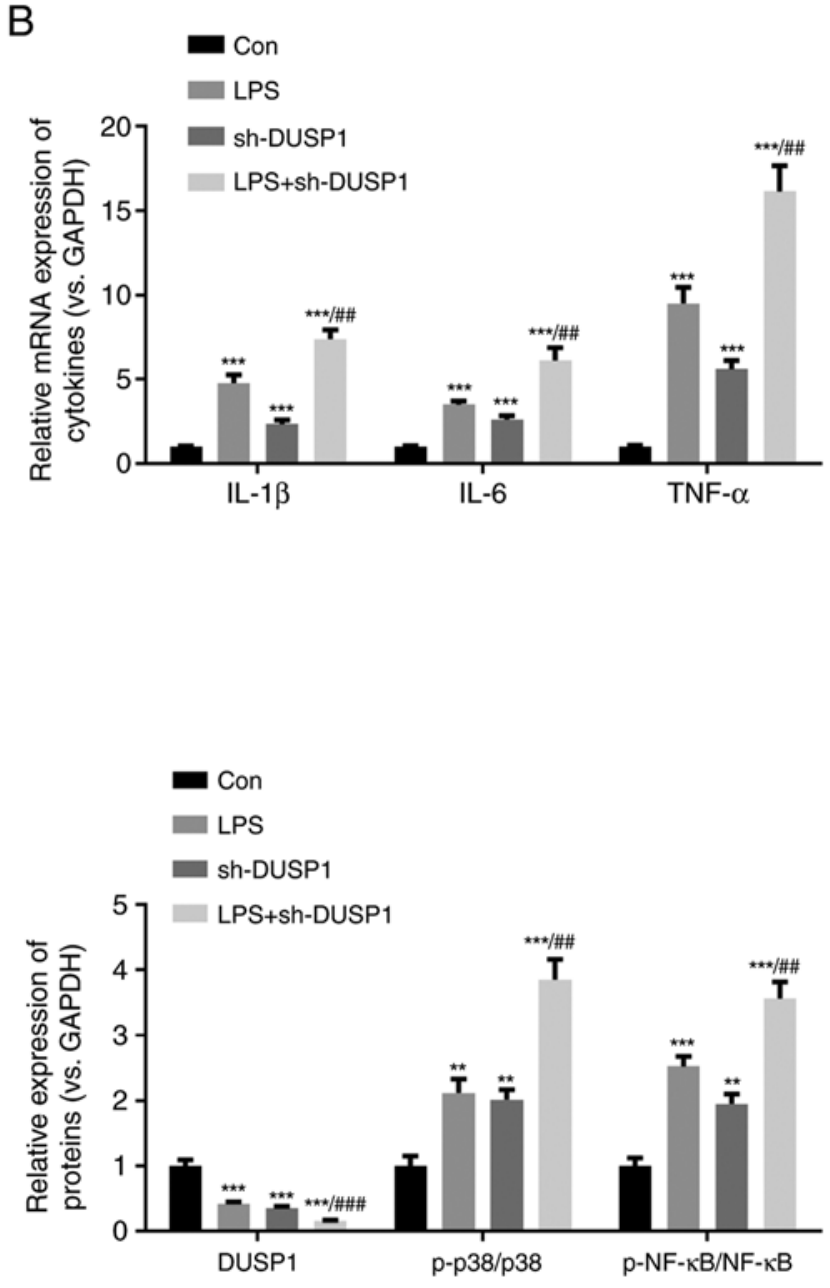

Figure 5. DUSP1 downregulation activates the MAPK-p38-NF- $\kappa$ B pathway. A cell model of downregulated DUSP1 was constructed by transfecting sh-DUSP1 into H9C2 cells, which were then treated with LPS $(10 \mu \mathrm{g} / \mathrm{ml})$ for $24 \mathrm{~h}$. (A) Western blot analysis was used to detect DUSP1 expression. (B and C) Reverse transcription-quantitative PCR was used to detect the expressional changes of inflammatory cytokines (including IL-1 $\beta$, IL-6 and TNF- $\alpha$ ). (C) Western blot analysis was performed to analyze the difference in expression of DUSP1, MAPK-p38 and NF- $\kappa \mathrm{B} .{ }^{* *} \mathrm{P}<0.01$ and ${ }^{* * *} \mathrm{P}<0.001$ vs. control (con). ${ }^{\# \#} \mathrm{P}<0.01$ and

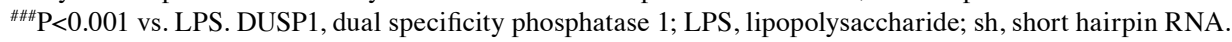

the luciferase activity of the DUSP1 wild-type (DUSP1-WT) group was significantly decreased following co-transfection with miR-101-3p mimics, while the luciferase activity of the DUSP1 mutant (DUSP1-MT) group exhibited no significant change (Fig. 4B). Furthermore, RT-qPCR, IHC and western blot analysis were performed to detect the levels of DUSP1, MAPK-p38 and NF- $\kappa \mathrm{B}$ in cardiac tissues. Compared with the sham group, DUSP1 expression was significantly downregulated in the LPS group (Fig. 4C-E), while the levels of phosphorylated MAPK-p38 and NF- $\kappa$ B were upregulated (Fig. 4E). However, the inhibition of miR-101-3p enhanced the expression of DUSP1, while downregulating the expression of phosphorylated MAPK-p38 and NF- $\kappa B$ (Fig. 4C-E). These data indicated that miR-101-3p may exerted its effects in SIC by targeting DUSP1.

Downregulation of DUSP1 activates the MAPK-p38 and $N F-\kappa B$ pathways. MAPK-p38 and NF- $\kappa \mathrm{B}$ are important signaling pathways associated with myocardial cell injury (6).
In the present study, to further verify the function of DUSP1, a cell model using H9C2 cells in which DUSP1 was downregulated was constructed (Fig. 5A). Furthermore, the H9C2 cells were stimulated with LPS. The results revealed that compared with the control group, both the LPS and sh-DUSP1 groups exhibited a significantly increased expression of inflammatory cytokines, increased levels of phosphorylated MAPK-p38 and NF- $\kappa \mathrm{B}$, and decreased levels of DUSP1 (Fig. 5B and C). Moreover, compared with the LPS group, the levels of inflammatory cytokines and phosphorylated MAPK-p38 and NF- $\mathrm{kB}$ were further enhanced in the LPS + sh-DUSP1 group. Hence, DUSP1 also plays a role in controlling the inflammatory response in $\mathrm{H} 9 \mathrm{C} 2$ cells by modulating the MAPK-p38 and NF-kB pathways.

Downregulation of miR-101-3p protects against LPS-induced myocardial cell injury dependently via DUSPI. To further examine the effects of miR-101-3p on targeting the DUSP1/MAPK-p38/NF-кB pathway in myocardial injury, the 


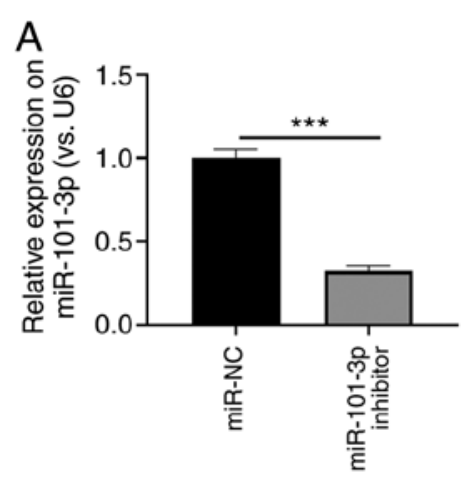

B

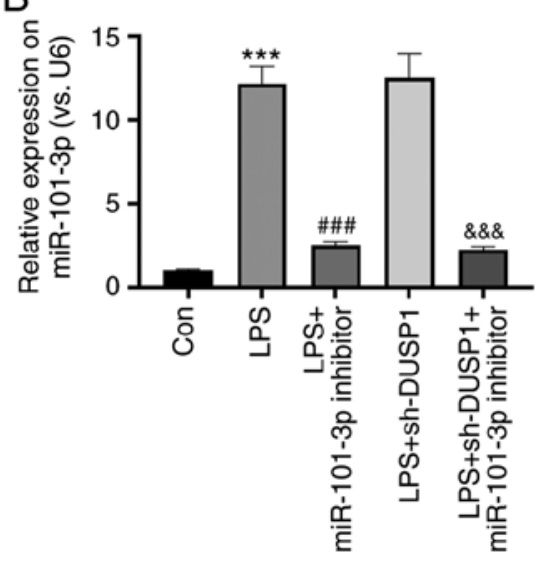

C

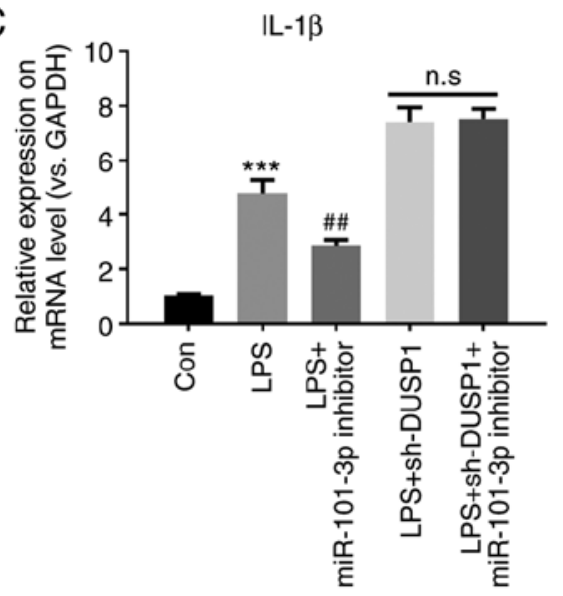

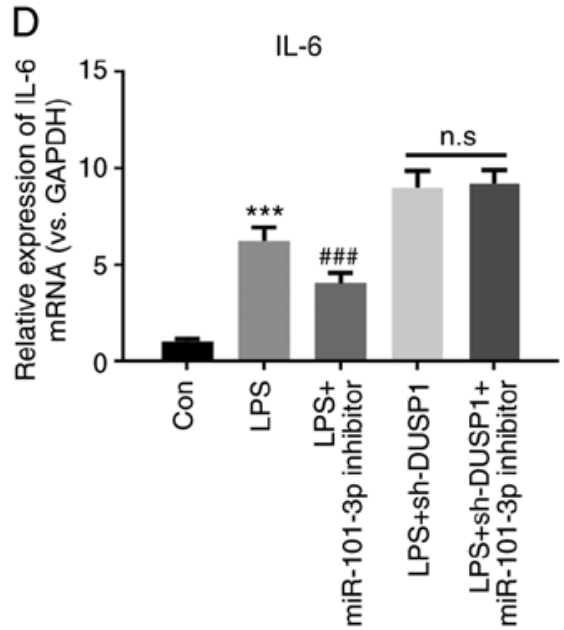

$\mathrm{F}$
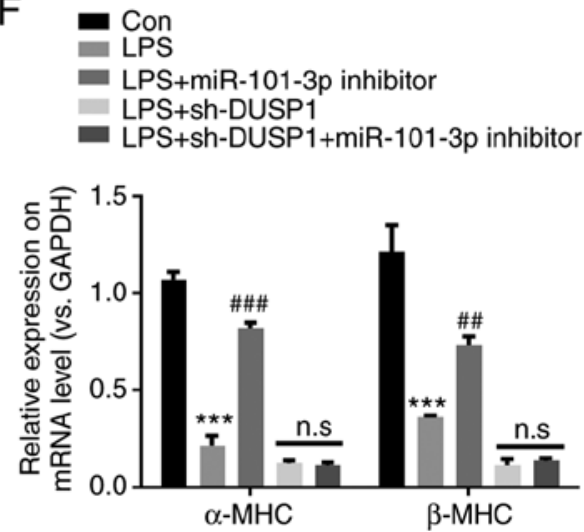

E

TNF- $\alpha$

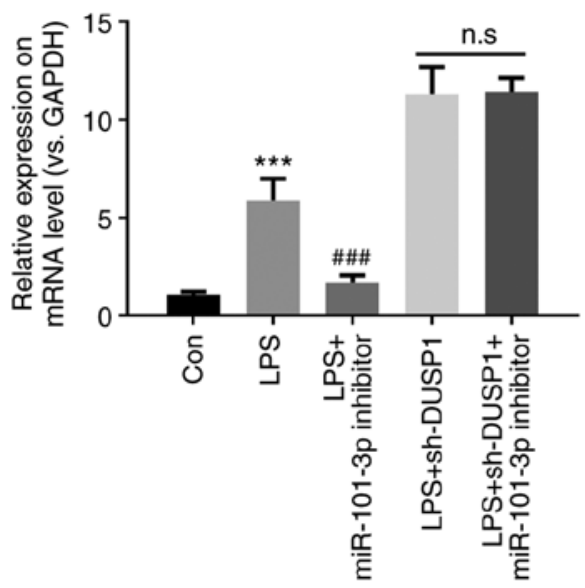

G
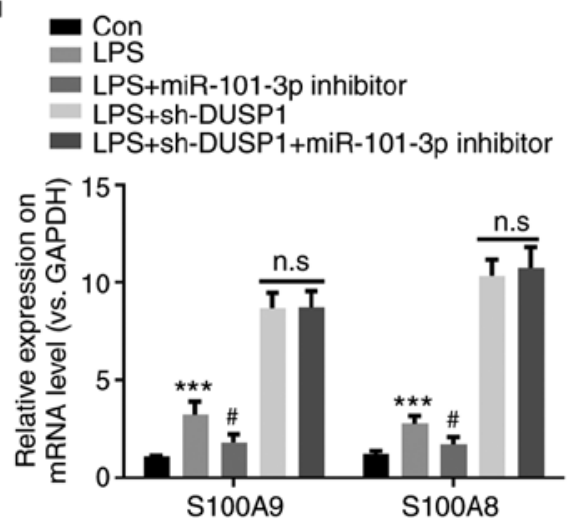

Figure 6. Downregulation of miR-101-3p protects against LPS-induced myocardial cell injury dependently via DUSP1. H9C2 cells transfected with sh-DUSP1 and/or miR-101-3p were treated with LPS (10 $\mu \mathrm{g} / \mathrm{ml})$. (A-G) Reverse transcription-quantitative PCR was used to detect the expression of (A and B) miR-101-3p, (C) IL-1 $\beta$, (D) IL-6, (E) TNF- $\alpha$, (F) $\alpha$-MHC and $\beta$-MHC, (G) S100A8 and S100A9. n.s, not significant $(\mathrm{P}>0.05) ;{ }^{* * * *} \mathrm{P}<0.001$ vs. con. ${ }^{~} \mathrm{P}<0.05$; ${ }^{\# \#} \mathrm{P}<0.01$ and ${ }^{\# \# \# P} \mathrm{P}<0.001$ vs. LPS. DUSP1, dual specificity phosphatase 1; LPS, lipopolysaccharide; sh, short hairpin RNA; MHC, myosin heavy chain; miR, microRNA.

H9C2 cells transfected with sh-DUSP1 and/or miR-101-3p were stimulated with LPS. The results revealed that miR-101-3p expression was significantly suppressed by miR-101-3p inhibitors with or without LPS stimulation ( $\mathrm{P}<0.05$; Fig. 6A and $\mathrm{B})$. RT-qPCR was then performed to detect the expression levels of IL-1 $\beta$, IL-6, TNF- $\alpha, \alpha-M H C, \beta-M H C$, S100A8 and S100A9. The results revealed that compared with the control group, the expression of IL- $1 \beta$, IL- 6 , TNF- $\alpha$, S100A 8 and S100A9 in the LPS group was significantly upregulated, while the levels $\alpha$-MHC and $\beta$-MHC were significantly downregulated $(\mathrm{P}<0.05$; Fig. 6C-G). Additionally, compared with the LPS group, the levels of IL-1 $\beta$, IL-6, TNF- $\alpha$, S100A8 and S100A9 were downregulated, while those of $\alpha-\mathrm{MHC}$ and $\beta-\mathrm{MHC}$ were significantly upregulated following transfection with miR-101-3p inhibitors $(\mathrm{P}<0.05$; Fig. 6C-G). However, the expression levels of IL-1 $\beta$, IL-6, TNF- $\alpha$, S100A8, S100A9, $\alpha$-MHC and $\beta$-MHC in the LPS + sh-DUSP $1+\operatorname{miR}-101-3 p$ inhibitor group did not significantly differ compared with 
those in the LPS + sh-DUSP1 group (Fig. 6C-G). These data suggest that miR-101-3p exerts its effects dependently by suppressing DUSP1.

\section{Discussion}

The present study demonstrated that miR-101-3p expression was significantly elevated in SIC and was positively associated with the degree of systemic inflammatory response. Further experiments suggested that miR-101-3p activated the MAPK-p38 and NF- $\kappa$ B pathways by inhibiting DUSP1 in cardiomyocytes, thereby aggravating sepsis-induced myocardial injury.

Septic shock is a common and critical illness in clinical practice with a high mortality rate. To date, there is still a lack of clinical treatments with high efficacy and minimal side-effects. Bacterial infection is the most common cause of septic shock. As a bacterial product, LPS can directly or indirectly stimulate monocytes, polymorphonuclear neutrophils, endothelial cells and other myocardial cells to initiate the inflammatory process, produce proinflammatory cytokines and increase inflammatory response $(25,26)$. Cardiomyocytes also respond to sepsis during the early stage, which is closely associated with disease prognosis. In particular, SIC, which is a highly monitored disease in the intensive care unit, is characterized by myocardial contractile function impairment, cardiac enlargement, decreased ejection fraction, poor response to volumetric load contraction and decreased peak contraction pressure/end-systolic volume ratio $(27,28)$. Therefore, the pathophysiological mechanism of SIC has been the focus of studies.

Studies have indicated that miRNAs play a crucial role in the regulation of the SIC inflammatory response, accompanied by dynamic changes in miRNA expression. miRNAs, as novel biomarkers of sepsis, can be used for severity grading, early diagnosis, treatment response and prognosis evaluation of SIC. However, the effects of miRNAs on SIC have not been systematically studied (29). Wang et al demonstrated that miR-21-3p expression is increased in animal models of LPS-induced SIC, whereas inhibitors of miR-21-3p can significantly improve cardiac function and survival in SIC (30). However, knowledge of the role of miR-101-3p in sepsis is limited. The results of the present study demonstrated that miR-101-3p expression was significantly increased in patients with SIC and positively correlated with the expression of IL- $1 \beta$, IL- 6 and TNF- $\alpha$, and other inflammatory factors, but was negatively associated with cardiac function. This suggests that miR-101-3p plays a role in promoting disease progression in SIC and may also be an indicator for SIC diagnosis and prognosis.

As a mature form of miR-101, decreased levels of miR-101-3p have been shown to exert tumor suppressor functions in numerous malignant tumors, and to regulate proliferation, invasion and metastasis $(20,21)$. However, to the best of our knowledge, the role of miR-101-3p in inflammatory diseases has been rarely reported. For instance, Qin et al reported that in a rat model of ischemia-reperfusion injury, zinc exerted protective effects by suppressing miR-101-3p, which promoted oxidative stress by activating nuclear factor erythroid 2-related factor 2 and NF- $\mathrm{KB}$ levels in TM3 cells (31). In another study, miR-101-3p was shown to reduce joint swelling and the arthritic index in rats with rheumatoid arthritis by downregulating prostaglandin $\mathrm{G} / \mathrm{H}$ synthase 2 (21). Nevertheless, miR-101-3p expression has been shown to be significantly higher in heart transplant recipients with histologically verified acute cellular rejection (22), as well as in plasma of patients with AOSD compared with HCs (20). Thus, the effect of miR-101-3p in various diseases may differ. The results of the presents study demonstrated that miR-101-3p exerted significant protective effects against sepsis-induced myocardial injury by suppressing inflammation and apoptosis, suggesting that miR-101-3p may be a valuable therapeutic target for sepsis-induced myocardial injury.

DUSP1 exerts a significant effect on the anti-inflammatory environment. For example, DUSP1 can regulate the pro-inflammatory environment of head and neck squamous cell carcinoma, thereby preventing cancer progression; it can also act on dexamethasone to improve the anti-inflammatory effects of drugs (32). Moreover, a previous study demonstrated that miR-127 can mediate the polarization of macrophages and facilitate lung inflammation and injury by activating the JNK pathway and downregulating Bc16 or DUSP1 expression (33). Additionally, MKP-1 knockdown re-phosphorylates p38 and restores the capacity to translate TNF- $\alpha$ and IL-6 mRNAs (34). These results indicate that DUSP1 exerts anti-inflammatory effects by phosphorylating and inactivating members of the MAPK family. The present study found that miR-101-3p can directly bind to the 3'-UTR region of DUSP1, thereby suppressing DUSP1 in cardiac cells. Moreover, the downregulation of miR-101-3p significantly upregulated the DUSP1 levels and suppressed the activation of the MAPK-p38/NF- $\mathrm{KB}$ pathway. Furthermore, the present in vitro experiments indicated that the downregulation of DUSP1 led to a significant increase in the inflammatory response and MAPK-p38/NF- $\kappa B$ pathway activation. Furthermore, the protective effects of miR-101-3p inhibition were attenuated following the decreased expression of DUSP1. Therefore, the inhibition of miR-101-3p inhibits the inflammatory response of cardiomyocytes and reduces myocardial injury in SIC dependently by enhancing DUSP1 expression.

The two MHC isoforms identified in mammalian ventricles, $\alpha$-MHC and $\beta$-MHC, contribute to a large difference in heart rate (35). Moreover, $\alpha-\mathrm{MHC}$ is mainly found in embryonic cardiomyocytes, while $\beta$-MHC is mostly expressed in mature cardiomyocytes. The $\beta$-MHC protein content reflects the differentiation of MSCs into cardiomyocytes to a certain extent, and its gene expression level is one of the important indicators for observing the differentiation of MSCs into mature cardiomyocytes (36). A previous study found that the upregulation of $\alpha-\mathrm{MHC}$ and $\beta$-MHC promoted the cardiac differentiation of mouse embryonic stem cells (37). Furthermore, in a model of LPS-induced sepsis, $\alpha$-MHC and $\beta-M H C$ were shown to both be significantly downregulated (38), which was also identified in the present study. The present study also found that the inhibition of miR-101-3p promoted the $\alpha$-MHC and $\beta$-MHC levels in the myocardial tissues and cardiomyocytes.

The S100 subfamily which belongs to the EF-hand family has been found to play a vital role in modulating heart functions (39). Notably, several S100 members, including S100A8, S100A9, S100A11 and S100A12 are proved as 
biomarkers in sepsis $(40,41)$. In addition, the downregulation of the S100 subfamily can exert protective effects against sepsis. For instance, pentamidine has been shown to ameliorate cecal ligation and puncture (CLP)-induced brain damage by mitigating gliosis and $\mathrm{S} 100 \mathrm{~B} / \mathrm{RAGE} / \mathrm{NF}-\kappa \mathrm{B}$ pathway activation (42). Alamandine has been shown to attenuate sepsis-induced S100A8 and S100A9 expression by suppressing MAPKs (38). The present study also found that S100A8 and S100A9 were overexpressed in the heart tissues and cardiomyocytes subjected to LPS. However, miR-101-3p inhibition markedly suppressed S100A8 and S100A9 and MAPK-p38 activation. Hence, the data further elucidated the underlying mechanisms of the protective effects of the inhibition of miR-101-3p against sepsis-induced heart injury.

In conclusion, the present study demonstrated that the inhibition of the miR-101-3p pathway can effectively inhibit the inflammatory response and myocardial cell damage in SIC, improve the cardiac function of SIC and play a role in myocardial protection. The mechanism involved may that the inhibition of miR-101-3p suppresses the SIC-induced inflammatory response by targeting the MAPK-p38/NF- $\mathrm{B}$ pathway via DUSP1. These findings also suggested that the inhibition of miR-101-3p may be a potential important target for the treatment and diagnosis of SIC.

\section{Acknowledgements}

Not applicable.

\section{Funding}

No funding was received.

\section{Availability of data and materials}

The datasets used and analyzed during the current study are available from the corresponding author on reasonable request.

\section{Authors' contributions}

FH had full access to all of the data in the study and all authors take responsibility for the integrity of the data and the accuracy of the data analysis. YX and LT were involved in the acquisition, analysis, or interpretation of the data. YX, JC and DC conducted RT-qPCR, H\&E staining and the related data analysis, and they were involved in the drafting of the manuscript. YX and WW were involved in performing the statistical analysis. All authors read and approved the final manuscript.

\section{Ethics approval and consent to participate}

The present study was approved by the Ethics Committee of the Affiliated Hospital of Southwest Medical University. Prior to the study, all participants signed informed consent forms and agreed to the use of their samples in this scientific research. The animal experiments were approved by the Ethics Committee of the Affiliated Hospital of Southwest Medical University. All experimental procedures were performed in accordance with the National Institutes of Health Guidelines for the Care and Use of Laboratory Animals.

\section{Patient consent for publication}

Not applicable.

\section{Competing interests}

The authors declare that they have no competing interests.

\section{References}

1. Frencken JF, Donker DW, Spitoni C, Koster-Brouwer ME, Soliman IW, Ong DSY, Horn J, van der Poll T, van Klei WA, Bonten MJM and Cremer OL: Myocardial injury in patients with sepsis and its association with long-term outcome. Circ Cardiovasc Qual Outcomes 11: e004040, 2018.

2. Lv X and Wang H: Pathophysiology of sepsis-induced myocardial dysfunction. Mil Med Res 3: 30, 2016.

3. Rudiger A and Singer M: Mechanisms of sepsis-induced cardiac dysfunction. Crit Care Med 35: 1599-1608, 2007.

4. Cao C, Zhang Y, Chai Y, Wang L, Yin C, Shou S and Jin H: Attenuation of sepsis-induced cardiomyopathy by regulation of microRNA-23b is mediated through targeting of MyD88-mediated NF- $\kappa$ B activation. Inflammation 42: 973-986, 2019.

5. Ouyang MZ, Zhou D, Zhu Y, Zhang M and Li L: The inhibition of MyD88 and TRIF signaling serve equivalent roles in attenuating myocardial deterioration due to acute severe inflammation. Int J Mol Med 41: 399-408, 2018.

6. Zeng M, Zhang B, Li B, Kan Y, Wang S, Feng W and Zheng X: Adenosine attenuates LPS-induced cardiac dysfunction by inhibition of mitochondrial function via the ER pathway. Evid Based Complement Alternat Med 2019: 1832025, 2019.

7. Chen S and Fan B: Myricetin protects cardiomyocytes from LPS-induced injury. Myricetin schützt Kardiomyozyten vor LPS-induzierten Verletzungen. Herz 43: 265-274, 2018.

8. Zheng Z, Ma H, Zhang X, Tu F, Wang X, Ha T, Fan M, Liu L, $\mathrm{Xu} \mathrm{J}, \mathrm{Yu} \mathrm{K}$, et al: Enhanced glycolytic metabolism contributes to cardiac dysfunction in polymicrobial sepsis. J Infect Dis 215: 1396-1406, 2017.

9. Qiu Z, He Y, Ming H, Lei S, Leng $Y$ and Xia ZY: Lipopolysaccharide (LPS) aggravates high glucose- and hypoxia/ reoxygenation-induced injury through activating ROS-dependent NLRP3 inflammasome-mediated pyroptosis in H9C2 cardiomyocytes. J Diabetes Res 2019: 8151836, 2019.

10. Wancket LM, Frazier WJ and Liu Y: Mitogen-activated protein kinase phosphatase (MKP)-1 in immunology, physiology, and disease. Life Sci 90: 237-248, 2012.

11. Boutros T, Chevet E and Metrakos P: Mitogen-activated protein (MAP) kinase/MAP kinase phosphatase regulation: Roles in cell growth, death, and cancer. Pharmacol Rev 60: 261-310, 2008.

12. Low HB and Zhang Y: Regulatory roles of MAPK phosphatases in cancer. Immune Netw 16: 85-98. 2016.

13. Hoppstädter J and Ammit AJ: Role of dual-specificity phosphatase 1 in glucocorticoid-driven anti-inflammatory responses. Front Immunol 10: 1446, 2019.

14. Kristiansen M, Hughes R, Patel P, Jacques TS, Clark AR and Ham J: Mkp1 is a c-Jun target gene that antagonizes JNK-dependent apoptosis in sympathetic neurons. J Neurosci 30: 10820-10832, 2010.

15. Rastogi R, Jiang Z, Ahmad N, Rosati R, Liu Y, Beuret L, Monks R, Charron J, Birnbaum MJ and Samavati L: Rapamycin induces mitogen-activated protein (MAP) kinase phosphatase-1 (MKP-1) expression through activation of protein kinase $\mathrm{B}$ and mitogen-activated protein kinase kinase pathways. J Biol Chem 288: 33966-33977, 2013.

16. Catalanotto C, Cogoni C and Zardo G: MicroRNA in control of gene expression: An overview of nuclear functions. Int J Mol Sci 17: 1712, 2016

17. Wang $\mathrm{X}$ and $\mathrm{Yu} \mathrm{Y}$ : MiR-146b protect against sepsis induced mice myocardial injury through inhibition of Notch1. J Mol Histol 49: 411-417, 2018.

18. Wu X, Zhou J, Wu Z, Chen C, Liu J, Wu G, Zhai J, Liu F and Li G: miR-101-3p suppresses HOX transcript antisense RNA (HOTAIR)-induced proliferation and invasion through directly targeting SRF in gastric carcinoma cells. Oncol Res 25: $1383-1390,2017$ 
19. Li CY, Xiong DD, Huang CQ, He RQ, Liang HW, Pan DH, Wang HL, Wang YW, Zhu HW and Chen G: Clinical value of miR-101-3p and biological analysis of its prospective targets in breast cancer: A study based on the cancer genome atlas (TCGA) and bioinformatics. Med Sci Monit Med Sci Monit 23: 1857-1871, 2017.

20. Hu Q, Gong W, Gu J, Geng G, Li T, Tian R, Yang Z, Zhang H, Shao L, Liu T, et al: Plasma microRNA profiles as a potential biomarker in differentiating adult-onset still's disease from sepsis. Front Immunol 9: 3099, 2019.

21. Wei Q, Lv F, Zhang H, Wang X, Geng Q, Zhang X, Li T, Wang S, Wang Y and Cui Y: MicroRNA-101-3p inhibits fibroblast-like synoviocyte proliferation and inflammation in rheumatoid arthritis by targeting PTGS2. Biosci Rep 40: BSR20191136, 2020.

22. Sukma Dewi I, Hollander Z, Lam KK, McManus JW, Tebbutt SJ, Ng RT, Keown PA, McMaster RW, McManus BM, Gidlöf O and Öhman J: Association of serum MiR-142-3p and MiR-101-3p levels with acute cellular rejection after heart transplantation. PLoS One 12: e0170842, 2017.

23. Chen L, Liu P, Feng X and Ma C: Salidroside suppressing LPS-induced myocardial injury by inhibiting ROS-mediated $\mathrm{PI} 3 \mathrm{~K} / \mathrm{Akt} / \mathrm{mTOR}$ pathway in vitro and in vivo. J Cell Mol Med 21: 3178-3189, 2017.

24. Livak KJ and Schmittgen TD: Analysis of relative gene expression data using real-time quantitative PCR and the 2(-Delta Delta C(T)) method. Methods 25: 402-408, 2001.

25. Jiang Q, Chen J, Long X, Yao X, Zou X, Yang Y, Huang G and Zhang H: Phillyrin protects mice from traumatic brain injury by inhibiting the inflammation of microglia via PPAR $\gamma$ signaling pathway. Int Immunopharmacol 79: 106083, 2020.

26. Kakihana Y, Ito T, Nakahara M, Yamaguchi K and Yasuda T: Sepsis-induced myocardial dysfunction: Pathophysiology and management. J Intensive Care 4: 22, 2016.

27. Tan Y, Chen S, Zhong J, Ren J and Dong M: Mitochondrial injury and targeted intervention in septic cardiomyopathy. Curr Pharm Des 25: 2060-2070, 2019

28. De Kock I, Van Daele C and Poelaert J: Sepsis and septic shock: Pathophysiological and cardiovascular background as basis for therapy. Acta Clin Belg 65: 323-329, 2010.

29. Li HM, Li KY, Xing Y, Tang XX, Yang DM, Dai XM, Lu DX and Wang HD: Phenylephrine attenuated sepsis-induced cardiac inflammation and mitochondrial injury through an effect on the PI3K/Akt signaling pathway. J Cardiovasc Pharmacol 73 : 186-194, 2019.

30. Wang H, Bei Y, Shen S, Huang P, Shi J, Zhang J, Sun Q, Chen Y, Yang Y, Xu T, et al: miR-21-3p controls sepsis-associated cardiac dysfunction via regulating SORBS2. J Mol Cell Cardiol 94: 43-53, 2019.

31. Qin Z, Zhu K, Xue J, Cao P, Xu L, Xu Z, Liang K, Zhu J and Jia R: Zinc-induced protective effect for testicular ischemia-reperfusion injury by promoting antioxidation via microRNA-101-3p/Nrf2 pathway. Aging (Albany NY) 11: 9295-9309, 2019.
32. Zhang X, Hyer JM, Yu H, D'Silva NJ and Kirkwood KL: DUSP1 phosphatase regulates the proinflammatory milieu in head and neck squamous cell carcinoma. Cancer Res 74: 7191-7197, 2014.

33. Ying H, Kang Y, Zhang H, Zhao D, Xia J, Lu Z, Wang H, Xu F and Shi L: MiR-127 modulates macrophage polarization and promotes lung inflammation and injury by activating the JNK pathway. J Immunol 194: 1239-1251, 2015.

34. Brudecki L, Ferguson DA, McCall CE and El Gazzar M: Mitogen-activated protein kinase phosphatase 1 disrupts proinflammatory protein synthesis in endotoxin-adapted monocytes. Clin Vaccine Immunol 20: 1396-1404, 2013.

35. Kawai M, Karam TS, Michael JJ, Wang L and Chandra M: Comparison of elementary steps of the cross-bridge cycle in rat papillary muscle fibers expressing $\alpha$ - and $\beta$-myosin heavy chain with sinusoidal analysis. J Muscle Res Cell Motil 37: 203-214, 2016.

36. Orlic D, Kajstura J, Chimenti S, Jakoniuk I, Anderson SM, Li B, Pickel J, McKay R, Nadal-Ginard B, Bodine DM, et al: Bone marrow cells regenerate infarcted myocardium. Nature 410: 701-705, 2001.

37. Lu Q, Liu Y, Wang Y, Wang W, Yang Z, Li T, Tian Y, Chen P, Ma K, Jia Z and Zhou C: Rapamycin efficiently promotes cardiac differentiation of mouse embryonic stem cells. Biosci Rep 37: BSR20160552, 2017

38. Li P, Chen XR, Xu F, Liu C,Li C, Liu H, Wang H, Sun W, Sheng YH and Kong XQ: Alamandine attenuates sepsis-associated cardiac dysfunction via inhibiting MAPKs signaling pathways. Life Sci 206: 106-116, 2018.

39. Xiao X, Yang C, Qu SL, Shao YD, Zhou CY, Chao R, Huang L and Zhang C: S100 proteins in atherosclerosis. Clin Chim Acta 502: 293-304, 2020.

40. Huang $\mathrm{H}$ and Tu L: Expression of S100 family proteins in neonatal rats with sepsis and its significance. Int J Clin Exp Pathol 8: 1631-1639, 2015.

41. Tosson AMS, Glaser K, Weinhage T, Foell D, Aboualam MS, Edris AA, El Ansary M, Lotfy S and Speer CP: Evaluation of the S100 protein A12 as a biomarker of neonatal sepsis. J Matern Fetal Neonatal Med 33: 2768-2774, 2020.

42. Huang L, Zhang L, Liu Z, Zhao S, Xu D, Li L, Peng Q and Ai Y: Pentamidine protects mice from cecal ligation and puncture-induced brain damage via inhibiting S100B/RAGE/NF- $\kappa$ B Biochem Biophys Res Commun 517: 221-226, 2019.

is work is licensed under a Creative Commons Attribution-NonCommercial-NoDerivatives 4.0 International (CC BY-NC-ND 4.0) License. 